\title{
Energy spectra of quasiperiodic systems via information entropy
}

\author{
Enrique Maciá* and Francisco Domínguez-Adame \\ Departamento de Física de Materiales, Facultad de Físicas, Universidad Complutense, E-28040 Madrid, Spain \\ Angel Sánchez \\ Escuela Politécnica Superior, Universidad Carlos III de Madrid, c. Butarque 15, E-28911 Leganés, Madrid, Spain
}

(Received 16 May 1994)

\begin{abstract}
We study the relationship between the electronic spectrum structure and the configurational order of onedimensional quasiperiodic systems. We take the Fibonacci case as a specific example, but the ideas outlined here may be useful to accurately describe the energy spectra of general quasiperiodic systems of technological interest. Our main result concerns the minimization of the information entropy as a characteristic feature associated with quasiperiodic arrangements. This feature is shown to be related to the ability of quasiperiodic systems to encode more information, in the Shannon sense, than periodic ones. In the conclusion we comment on interesting implications of these results on further developments on the issue of quasiperiodic order.
\end{abstract}

PACS number(s): 61.44. $+\mathrm{p}, 65.50 .+\mathrm{m}, 71.20 .-\mathrm{b}, 05.90 .+\mathrm{m}$

The notion of quasiperiodic order (QPO) has been given a very different status in physics, depending on the perspective adopted by authors working on different fields. From a theoretical point of view, the necessity to achieve a good understanding of the transport properties of disordered systems has led a number of researchers to address the issue of quasiperiodicity as somewhat intermediate between periodic order and purely random disorder [1]. From this perspective the notion of QPO assumes a subsidiary role as a mere way to describe the conceptual transition from periodic order to randomness. On the other side, from a practical viewpoint, it has been progressively realized, starting from the works by Merlin et al. [2] and Todd et al. [3] on quasiperiodic superlattices, that electronic devices with this particular kind of structure offer interesting possibilities for technological applications. In this case the study of those characteristic features directly related to the underlying QPO becomes interesting in its own right.

The electronic energy spectra of quasiperiodic systems has been extensively studied in the framework of real-space renormalization group techniques [4-6], where the original system is decoupled into a certain number of minor subsystems according to a given, so called, blocking scheme (BS). To date, two BSs have been proved to be remarkably useful in describing on-site and transfer models within the tight-binding approximation [5]. Less attention has been paid, however, to more general mixed models, in which both diagonal and off-diagonal terms are taken into account in the system Hamiltonian. Such models are, of course, more appropriate in order to describe realistic systems, where interactions between their different constituents depend on their specific chemical nature.

In this Rapid Communication we report on two general results. On one side, we show that the BS originally proposed to study on-site models can be extended to describe mixed models, hence indicating its applicability to a wide

\footnotetext{
*Also at Instituto de Estudios Interdisciplinares, El Guijo, Z4 Galapagar, E-28260 Madrid, Spain.
}

variety of quasiperiodic systems. On the other side, we show that this fact is related to the behavior of the information entropy function associated with these systems. In spite of our main conclusions holding for general one-dimensional systems exhibiting QPO, in this work we focus on Fibonacci arrangements for the sake of illustration. The reasons for this particular choice are twofold. In the first place, energy spectra corresponding to Fibonacci systems have been experimentally probed in a variety of situations, confirming that Fibonacci arrangements exhibit spectra with a hierarchy of splitting minibands displaying self-similar patterns [7-10], even when relativistic effects are taken into account [11]. In addition, we have shown recently that this fractal structure of the energy spectrum has relevant consequences on the dc conductance of the system [12]. In the second place, the widespread appearance of ordering patterns and structures based on the Fibonacci sequence in many biological systems is currently well established $[13,14]$. Therefore the basic ideas inspiring our work can be applied to a large variety of interesting systems.

Let us start by introducing our model Hamiltonian. We shall consider a system describing a binary substitutional alloy in which the constituent atoms are arranged according to the Fibonacci sequence. In general, a Fibonacci chain of order $N$ is generated from two basic units $A$ and $B$ by successive applications of the substitution rule $A \rightarrow A B$ and $B \rightarrow A$ yielding a sequence of the form $A B A A B A B A \cdots$. This sequence comprises $F_{N-1}$ elements $A$ and $F_{N-2}$ elements $B$, $F_{l}$ being the $l$ th Fibonacci number given by the recurrent law $F_{l}=F_{l-1}+F_{l-2}$ with the initial values $F_{0}=F_{1}=1$. In an actual alloy the hopping integrals describing the interaction between nearest-neighbor atoms would take different values depending upon the chemical nature of atomic species. In order to take into account this fact, we propose a general mixed model in the one-electron approximation. The interaction of an electron with the host atoms is described by means of $\delta$-function potentials. This is not a serious limitation since the $\delta$ function is a good approximation to more realistic short-ranged potentials [15]. Therefore we consider the following Schrödinger equation in units such that $\hbar=m=1$ : 


$$
\left[-\frac{1}{2} \frac{d^{2}}{d x^{2}}-\sum_{n} \lambda_{n} \delta(x-n d)\right] \psi(x)=E \psi(x),
$$

where we are dealing with equally spaced atomic positions, $d$ being the nearest-neighbor atomic distance. We allow the potential strength $\lambda_{n}$ to take on two values, $\lambda_{A}$ and $\lambda_{B}$, arranged according to the Fibonacci sequence. Hereafter we restrict ourselves to attractive potentials $\left(\lambda_{n}>0\right)$ and take $\lambda_{A}=1$ without loss of generality. Expressing the electron wave function as a linear combination of atomic orbitals $\quad \psi(x)=\Sigma_{n} C_{n} \phi_{n}(x-n d)$, where $\phi_{n}(x-n d)$ $=\sqrt{\lambda_{n}} \exp \left(-\lambda_{n}|x-n d|\right)$ is the normalized eigenfunction of a $\delta$ function placed at $x=n d$, and neglecting the overlap involving three different centers, we obtain the following tightbinding equation [12]:

$$
\left(E-\epsilon_{n}\right) C_{n}=t_{n, n+1} C_{n+1}+t_{n, n-1} C_{n-1},
$$

where the on-site energies and the hopping integrals are given by

$$
\begin{gathered}
\epsilon_{n}=-\frac{1}{2} \lambda_{n}^{2}, \\
t_{n, n \pm 1}=-\sqrt{\lambda_{n}^{3} \lambda_{n \pm 1}} \exp \left(-\lambda_{n \pm 1} d\right) .
\end{gathered}
$$

A complete description of the resulting electronic spectrum can be found elsewhere [12]. Here we shall merely give a brief summary of those results relevant to our purposes. In our numerical simulations we have studied in detail different realizations by varying the chain length $N$, the interatomic distance $d$, and the ratio $\alpha \equiv \lambda_{B} / \lambda_{A}$ which accounts for the chemical diversity of the alloy. In all cases considered we have observed a well differentiated tetrafurcation pattern of the energy spectrum, characterized by the presence of four main subbands separated by well-defined gaps. Inside each main subband the fragmentation scheme follows a trifurcation pattern in which each subband further trifurcates obeying a hierarchy of splitting from one to three subsubbands. In Fig. 1 we plot a typical resulting integrated density of states (IDOS) per unit length. Its staircase structure clearly shows the tetrafurcation of the spectrum as well as its selfsimilarity. At this point we shall exploit the capability of the IDOS to provide a link between the occupation of the various minibands appearing in the spectrum and the underlying atomic arrangement. In fact, since each atom in the chain contributes with one electronic state to the energy spectrum, the fraction of states is directly related to the heights of the characteristic steps appearing in the IDOS. In this way, we have measured the fraction of states appearing in the main subbands, $q_{i}$, for a wide range of both $\alpha$ and $d$ parameters. The measured heights yield the same values for all the model parameters considered. These values agree within an error less than $0.1 \%$ to $q_{a}=q_{c}=\tau^{3}, q_{b}=\tau^{4}$ and $q_{d}=\tau^{2}$, where $\tau=(\sqrt{5}-1) / 2$ is the inverse of the golden mean. These values completely agree with those obtained by Liu and Sritrakool for the IDOS corresponding to on-site models, which were interpreted in terms of a BS associated with the existence of isolated $A$ and $B$ atomic blocks and $A A$ diatomic blocks [5]. In that model, the hierarchical splitting of the spectrum is determined by short-range effects, and its overall

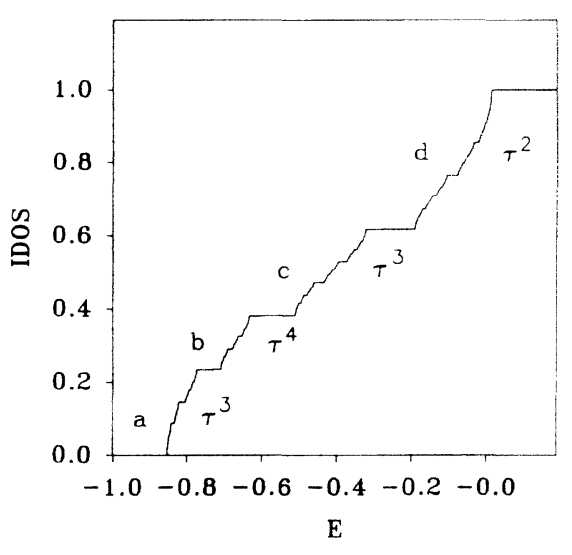

FIG. 1. Plot of the IDOS versus energy with model parameters given by $\alpha=0.75, d=1.5$, and $N=987$. The main subbands are labeled by $a, b, c$, and $d$ along with their respective fraction of states. Note that the level population exhibits characteristic features of a nonequilibrium system.

structure is due to the resonant coupling among states with nearly the same energy. As a result, the number of energy levels appearing at the first stage of the renormalization process determines the number of main subbands in the corresponding spectrum. Keeping this in mind, the convenience of a renormalization approach in our mixed model is justified by the following fact: we have observed that both the position and widths of the main subbands of the spectrum converge rapidly to stable values with increasing chain length. This behavior, which is independent of the chemical diversity of the alloy, has been referred to as asymptotic stability of the spectrum [12] and it seems to be a quite general property of both quasiperiodic [16] and homogeneously disordered alloys [17]. The asymptotic stability hence suggests that the number of main subbands in the spectrum is determined by short-range quantum effects, as it is required. Therefore, according to the renormalization group ideas just exposed, our obtained IDOS patterns indicate that we must block the original Fibonacci chain into a sequence of isolated $A$ and $B$ atomic blocks and diatomic $A A$ blocks in order to obtain the purported step heights. Thus we arrive at our first main result, namely, that a $\mathrm{BS}$, originally proposed to account for the energy spectrum of a very particular kind of system, can be extended to properly describe more complex and realistic quasiperiodic systems.

Now, this interesting result deserves some consideration, since it indicates that the electronic spectrum of these more general systems can still be accounted for by decimating the original chain in terms of isolated $A$ and $B$ atomic blocks and $A A$ diatomic blocks, even if one allows for a significative $A B$ interaction, as we do in our model. This result strongly suggests that we are facing a property associated with the QPO, rather than a feature attributable to the particular choice of the parameters describing the considered model. In this regard, we feel it has not been previously stressed in the literature that, for any arbitrary chain, quasiperiodic or not, there exist a manifold of possible BSs which may be considered in principle. Hence the question arises as to whether a particular scheme will be more appropriate than another in 
order to describe the energy spectrum splitting pattern. In the rest of this paper we shall show that, for systems exhibiting a QPO based on the application of a substitution rule, the more appropriate BSs are those which minimize their associated information entropy function, defined as follows.

Let us consider a general binary system composed of two different species, $A$ and $B$. Although we shall consider these species to be completely general in nature, we shall refer to them as "atoms" for convenience henceforth. According to the above presented numerical results, it does not seem to be necessary to consider blocks containing more than two atoms in the first stage of the renormalization process, within the weak-bound approximation. Then, the most complete BS to be considered should involve isolated $A$ and $B$ atomic blocks and $A A, A B, B A$, and $B B$ diatomic blocks. As stated before, there are many ways in which we can decouple the original chain in a series of atomic and diatomic blocks, each one producing a particular BS. Therefore we can assign to each particular BS a probability set of the form $\left\{p_{A}, p_{B}, p_{A A}, p_{A B}, p_{B A}, p_{B B}\right\}$, where $p_{j}$ is the probability of finding a given atomic or diatomic block along the blocked chain. In the thermodynamic limit these probabilities are obtained as $p_{j}=\lim \left(n_{j} / N\right)$, where $n_{j}$ is the number of $j$-type blocks and $N$ the total number of atoms in the chain. In this way, we can consider the set of possible BSs as a statistical ensemble in the usual sense and associate with it an information entropy function given by

$$
S=-k \sum_{j} p_{j} \ln p_{j}
$$

where $k$ is an appropriate constant. It then results that each possible BS can be properly characterized by its information entropy. Now, it is clear that the entropy function (5) will also depend on the kind of order exhibited by the alloy we are considering. Actually, it turns out that such dependence is very special for systems exhibiting QPO. Let us consider, as an example, the case of Fibonacci QPO. In order that the probability set $\left\{p_{j}\right\}$ can describe a Fibonacci sequence we must impose certain constraints onto the possible values of the different probabilities appearing in it, namely, these probabilities must satisfy the well-known Fibonacci limits, $\lim \left(n_{B} / n_{A}\right)=\tau$, and $\lim \left(n_{A} / n_{A A}\right)=\tau$, along with the normalization condition $p_{A}+p_{B}+2\left(p_{A A}+p_{A B}\right)=1$, which implies that $p_{A B}=p_{B A}$ and $p_{B B}=0$ in a Fibonacci chain. In doing so, we get the following relationships:

$$
\begin{gathered}
p_{A}=\tau^{3}\left(\tau-p_{A B}\right), \\
p_{B}=\tau^{2}-p_{A B}, \\
p_{A A}=\tau^{2}\left(\tau-p_{A B}\right),
\end{gathered}
$$

where, for convenience, we have left $p_{A B}$ as a free parameter. By inspection of expressions (6) we see that the condition $p_{j} \geqslant 0$ implies $0 \leqslant p_{A B} \leqslant \tau^{2}$ and that $p_{A}$ and $p_{B}$ cannot be simultaneously zero. As a consequence, the original Fibonacci chain cannot be decoupled into any series of diatomic $A B$ and $A A$ blocks alone. Thus we are led to the conclusion that certain BSs are prevented by the QPO exhibited by the system. Alternatively, it seems reasonable then

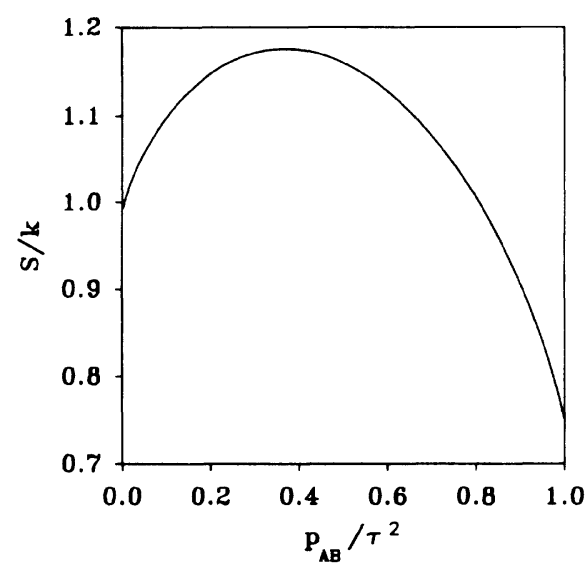

FIG. 2. Plot of the information entropy curve versus the probability $p_{A B}$. The appropriate blocking schemes accommodate to the minima of entropy function. See more details in the main text.

that certain BSs might be favored by the QPO. In order to prove this point we evaluate the information entropy function associated with the Fibonacci chain making use of the relations (6). In Fig. 2 we present its dependence on the probability $p_{A B}$. The entropy curve exhibits a characteristic maximum and two minima at the extreme points of its domain. The minimum at $p_{A B}=0$ corresponds to the level populations observed in our IDOS and it is related to the BS proposed by Liu and Sritrakool [5]. The minimum at $p_{A B}=\tau^{2}$ is associated with the consideration of three kinds of blocks as well, namely, $A$ isolated atomic blocks and $A A$ and $A B$ diatomic blocks. The corresponding level population can be obtained from (6) making use of the relationship $\tau^{n}+\tau^{n-1}=\tau^{n-2} \quad(n>2)$, and is given by the sequence $\tau^{5}: \tau^{2}: \tau^{6}: \tau^{5}: \tau^{2}$. No evidence has been observed in our numerical simulations for this pentafurcation pattern of the IDOS. The reason for this absence becomes clear from inspection of Fig. 3, where we show the behavior of the diatomic block energies, $E_{A A}$ and $E_{A B}$, as a function of the

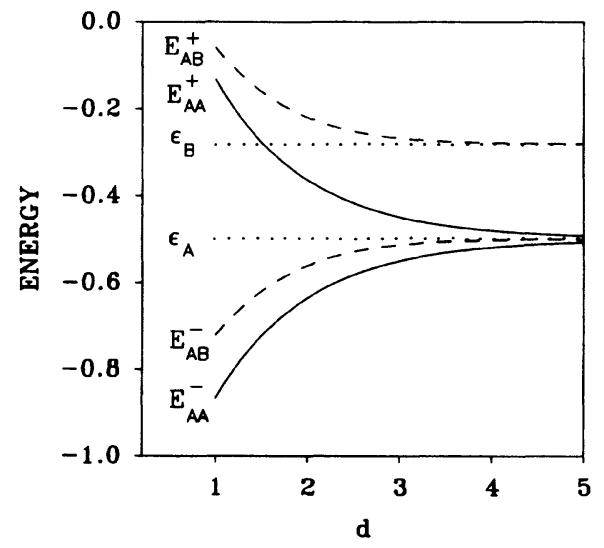

FIG. 3. Plot showing the behavior of diatomic block energies $E_{A A}^{ \pm}$(solid lines) and $E_{A B}^{ \pm}$(dashed lines) with interatomic distance $d$ for $\alpha=0.75$. Energies corresponding to isolated $A$ and $B$ atoms are also plotted for comparison (dotted lines). 
atomic distance for a fixed value of the chemical diversity, $\alpha$. In fact, we see that energy levels associated with the blocks $A A$ are always below the levels corresponding to the blocks $A B$, hence indicating that the $A B$ interaction is not strong enough to force all $B$ atomic levels to form $A B$ diatomic blocks in our model.

In this way we arrive at our second main result, namely, that the most favorable BSs for a mixed Fibonacci system are those which minimize their associated information entropy. Although this result has been obtained for a particular kind of QPO, we conjecture that the principle of minimum information entropy, as a criterion to properly choose the most appropriate BS, may hold for a broader class of quasiperiodic systems as well. The reasons sustaining our assumption rely on the following facts. In the first place, quasiperiodic systems do encode more information, in the sense of Shannon, than periodic or random ones, since the information content of a periodic chain is independent of length, whereas that of a quasiperiodic system increases with length. As a consequence, any quasiperiodic system will contain more information than a periodic one if it is sufficiently long, supporting our view that systems exhibiting QPO should more appropriately be described in terms of minima of the associated information entropy. In the second place, from the IDOS shown in Fig. 1 we can see that high energy levels are more populated than low energy ones at zero temperature. This remarkable feature also appears in the IDOS describing other aperiodic systems generated by the application of substitution rules, like Thue-Morse, period-doubling or RudinShapiro [18]. Therefore it is tempting to say that QPO describes far from thermodynamical equilibrium systems. The fabrication of one-dimensional quasicrystals exhibiting short Fibonacci stacking microstructure by means of nonequilibrium methods, such as rapid quenching from the melt [19] as well as the growth of Fibonacci superlattice heterostructures by molecular beam epitaxy [2], supports this point of view.
Now, it is accepted that the information entropy equals the thermodynamic entropy for equilibrium systems. There is no claim, however, that the information entropy represents the correct expression for the thermodynamic entropy of a system that is not in equilibrium (or that it does not) [20]. In this regard quasiperiodic systems could provide a suitable example of nonequilibrium systems for which the information entropy is quite different from its (vanishing) configurational entropy.

In summary, we have obtained valuable results concerning the relationship between the electronic spectrum structure and the information entropy content of a general Fibonacci system describing a binary alloy. Our results strongly support the view that QPO describes highly ordered systems able to encode more information than crystalline ones. By introducing a general approach to the study of quasiperiodic systems we provide a link between the atomic arrangement and the electronic structure displayed by the IDOS, indicating that the QPO describes systems far from equilibrium. Therefore the maximization entropy formalism, which has proved to be a good strategy of optimal prediction for both periodic and random systems, does not seem to work well for quasiperiodic ones. Finally, the general treatment introduced in this work can be extended, in a straightforward manner, in order to describe energy spectra of quasiperiodic systems other than the Fibonacci one. Work in this sense, regarding both electron and phonon energy spectra of systems displaying QPO, is currently in progress, and we expect to report on it elsewhere.

This work is partially supported by UCM under Project No. PR161/93-4811. A.S. is partially supported by DGICyT (Spain) through Project No. PB92-0248, and by the European Union Human Capital and Mobility Programme through Contract No. ERBCHRXCT930413. We thank M. V. Hernández for a critical reading of the manuscript.
[1] M. Kohmoto, L. P. Kadanoff, and C. Tang, Phys. Rev. Lett. 50, 1870 (1983).

[2] R. Merlin, K. Bajema, R. Clarke, F.-Y. Juang, and P. K. Bhattacharya, Phys. Rev. Lett. 55, 1768 (1985).

[3] J. Todd, R. Merlin, R. Clarke, K. M. Mohanty, and J. D. Axe, Phys. Rev. Lett. 57, 1157 (1986).

[4] Q. Niu and F. Nori, Phys. Rev. Lett. 57, 2057 (1986); Phys. Rev. B 42, 10329 (1990).

[5] Y. Liu and W. Sritrakool, Phys. Rev. B 43, 1110 (1991).

[6] G. Y. Oh and M. H. Lee, Phys. Rev. B 48, 12465 (1993).

[7] F. Laruelle and B. Etienne, Phys. Rev. B 37, 4816 (1988).

[8] M. Nakayama, H. Kato, and S. Nakashima, Phys. Rev. B 36, 3472 (1987).

[9] K. Kono, S. Nakada, Y. Narahara, and Y. Ootuka, J. Phys. Soc. Jpn. 60, 368 (1991).

[10] D. Tuet, M. Potemski, Y. Y. Wang, J. C. Maan, L. Tapfer, and K. Ploog, Phys. Rev. Lett. 66, 2128 (1991).
[11] F. Domínguez-Adame and A. Sánchez, Phys. Lett. A 159, 153 (1991).

[12] E. Maciá, F. Domínguez-Adame, and A. Sánchez, Phys. Rev. B 49, 9503 (1994).

[13] S. Douady and Y. Couder, Phys. Rev. Lett. 68, 2098 (1992).

[14] L. S. Levitov, Europhys. Lett. 14, 533 (1991).

[15] P. Erdös and R. C. Herndon, Helv. Phys. Acta 50, 109 (1977).

[16] M. Baake, D. Joseph, and P. Kramer, Phys. Lett. A 161, 153 (1991).

[17] A. Cordelli, G. Grosso, and G. P. Parravicini, Phys. Rev. B 48, 11567 (1993).

[18] J. M. Luck, Phys. Rev. B 39, 5834 (1989).

[19] L. Y. He, X. Z. Li, Z. Zhang, and K. H. Kuo, Phys. Rev. Lett. 61, 1116 (1988).

[20] M. S. Robertson, Statistical Thermophysics (Prentice-Hall, Englewood Cliffs, NJ, 1993). 